Mittlere Oerter der Vergleichsterne.

\begin{tabular}{|c|c|c|c|}
\hline$*$ & $\alpha 1900.0$ & $\delta$ I 900.0 & Autorität \\
\hline $\mathbf{I}$ & $2^{\mathrm{h}} 49^{\mathrm{m}} \times 8^{\mathrm{s}} \cdot 76$ & $+17^{\circ} 19^{\prime} 43^{\prime \prime} .8$ & AG. Berlin A. $78_{3}$ \\
\hline 2 & $249 \quad 23.40$ & $+20233^{6.9}$ & AG. Berlin B. 855 \\
\hline 3 & $249 \quad 43.4^{6}$ & +262823.7 & AG. Cambr. $15 \times 2$ \\
\hline
\end{tabular}

2. Beobachtungen über die Grösse und Helligkeit des Cometen.

$D$ ist der scheinbare Durchmesser des Cometen, $C$ die Schweiflänge, wobei, wenn zwei Zahlen gegeben sind, die erste die deutlich sichtbare Partie, die zweite die Länge bedeutet, bis zu welcher die schwächere Partie des Schweifes

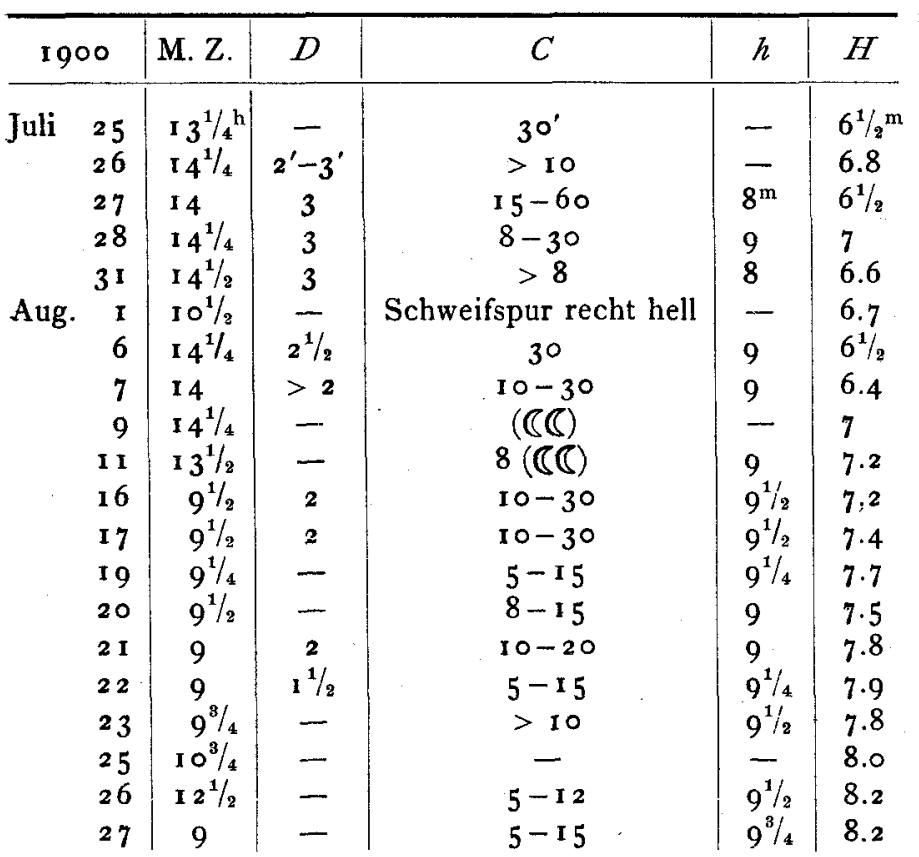

bei klarer Luft noch einigermaassen sicher verfolgt werden konnte; $h$ ist die Helligkeit des Kerns oder der kernähnlichen Lichtverdichtung im 6-zöll. Refractor, $H$ der Helligkeitseindruck des ganzen Cometen im kleinen Sucherfernrohr.

\begin{tabular}{|c|c|c|c|c|c|}
\hline 1900 & M. Z. & $D$ & $C$ & $h$ & $H$ \\
\hline Aug. $3^{\circ}$ & $9^{h}$ & - & $>8^{\prime}$ & $9 / 2 \mathrm{~m}$ & $8^{m} \cdot 3$ \\
\hline $3 I$ & 9 & $\mathbf{I}^{\prime}$ & $>8$ & $9^{1 / 2}$ & 8.5 \\
\hline Sept. 3 & $11 \frac{1}{4}$ & - & 12 & 10 & 8.7 \\
\hline 14 & 8 & - & Io? & IO & 9.2 \\
\hline 15 & $8 \%$ & - & & - & 9.2 \\
\hline I 6 & 8 & - & schwache & - & $9 \cdot 3$ \\
\hline 17 & 8 & - & Schweifspur & $91 / 2$ & 9.2 \\
\hline 19 & $93 / 4$ & - & & 10 & 9.5 \\
\hline 22 & $10 \%$ & - & & - & 9.5 \\
\hline 23 & 8 & - & - & - & 9.5 \\
\hline 25 & $8^{3} / 4$ & - & - & - & 9.7 \\
\hline 27 & $7 / 4$ & $11 / 2$ & - & I O. I I & 9.5 \\
\hline $3^{\circ}$ & 9 & $1 \%$ & & - & 10 \\
\hline Oct. I & I $11 / 4$ & - & & 一 & $10 \%$ \\
\hline 2 & I $11 / 4$ & 一 & vom Schweif & 一 & 10 \\
\hline 4 & $14{ }^{1 / 4}$ & - & nichts mehr zu & - & $10^{1 / 2}$ \\
\hline $\begin{array}{r}13 \\
16\end{array}$ & $\begin{array}{l}0 \\
9\end{array}$ & - & erkennen & - & $10 \%$ \\
\hline 24 & 9 & - & & - & $11^{3} / 4$ \\
\hline 28 & $7 \%$ & - & & - & $11 \%$ \\
\hline
\end{tabular}

F. Holetschek.

\title{
Periodische Veränderungen im Spectrum der Nova Persei.
}

Von dem interessanten Spectrum der Nova Persei habe ich eine Reihe photographischer Aufnahmen mit meinem I $0^{1 / 4}$ Reflector, theils mit einem I 0 -zölligen Objectivprisma theils mit einem Quarzspectrographen angefertigt. Bis heute kam ich nicht dazu, das interessante Material zu bearbeiten, eine eigenthümliche periodische Veränderung hat mich aber veranlasst, die folgende vorläufige Mittheilung zu veröffentlichen.

Mir ist zuerst eine plötzliche Veränderung des Spec. trums am 6. April aufgefallen. Ich habe mehrere, mit dem Objectivprisma gemachten Aufnahmen vom 31. März und 6. April verglichen und bemerkt, dass das continuirliche Spectrum am 6. April bedeutend schwächer geworden ist und auf dem schwachen Grunde die auffallend starken und breiten hellen linien besonders contrastreich auftraten. Die Schwächung des continuirlichen Spectrums habe ich der Lichtabnahme der Nova zugeschrieben, auffallend war aber, dass statt der hellen Wasserstofflinie $H \zeta(\lambda=3889)$ eine sehr starke, breite, helle Linie sichtbar war, welche ich oft in den planetarischen Nebeln gefunden habe, $(\lambda$ circa 3860 ). Diese Linie hatte die grösste Helligkeit, so dass sie entschieden heller war als $H \gamma$, was ich aus mit stehendem Uhrwerk aufgenommenen Spectren ganz sicher constatirte. Am 8. April (Objectivprisma) habe ich wieder das alte Spectrum, mit starkem continuirlichen Grunde, erhalten und statt der dicken Nebellinie war wieder $H \zeta$ sichtbar.

Die Helligkeit hat bedeutend zugenommen, die Aufnahme am 3 ז. März mit zwei Minuten Exposition war mit derjenigen vom 6. April mit Io Minuten gleich, und die Aufnahme yom 8. April mit zwei Minuten war bedeutend intensiver als die eben genannten.

Ich habe immer Io bis I 2 Aufnahmen gemacht von I bis 30 Minuten Exposition, so dass ein Vergleich leicht ausführbar ist.

Da bei der geringen Dispersion des Objectivprismas die Wasserstofflinie $H \zeta(\lambda=3889)$ und die Nebellinie $(\lambda=3860)$ sehr nahe zusammen fallen, habe ich die späteren Aufnahmen 
mit meinem Quarzspectrographen gemacht und gleichzeitig das Wasserstoffspectrum mitphotographirt.

Auf diesem Wege ist es mir gelungen, die vermuthete periodische Veränderung ganz sicher zu constatiren.

Am I I. April habe ich zwei Aufnahmen gemacht (20 Minuten und eine Stunde), welche derjenigen vom 6. April ähnlich sind, recht matter continuirlicher Grund, sehr starke Linien, welche beim Wasserstoff alle nach dem rothen Ende verschoben sind. Nebellinie entschieden auf der violetten Seite der künstlichen $H \zeta$-Linie, keine Coincidenz mit derselben, ausserdem - eine kräftige ultraviolette Linie (gegen $\lambda=34^{2}$ ) und eine schwächere (gegen $\lambda=339$ ), zusammen 15 bis 16 helle Linien.

Am 15. April (30 Minuten) sehr schwaches Bild wegen Wolken, ähnlich wie am Ir. April.

18. April. Zwei Aufnahmen (15 Min., 45 Min.). Das continuirliche Spectrum ist sehr stark, $H \zeta$ deutlich sichtbar, keine Spuren von der Nebel- und der ultravioletten Linie, wie am 31. März und 8. April. Das Aussehen des Spectrums ist so abweichend von demjenigen am 11. April, dass ich auf den ersten Blick an eine Verwechselung der Nova mit einem gewöhnlichen Stern glaubte, bis ich die hellen Wasserstofflinien mit einer Lupe deutlich gesehen hatte.

21. A pril. Wieder das Spectrum vom 11. April.

22. April. Ebenso, beide schöne kräftige Aufnahmen.
25. April. Aehnliche, sehr schwache Aufnahme, ultraviolette Linie (342) deutlich sichtbar.

27. April. Zwei Aufnahmen (so Min., eine Stunde). Starkes continuirliches Spectrum, von welchem die hellen Wasserstofflinien kaum abstechen. Nebellinie scheint im Uebergangsstadium zu sein, ultraviolette Linien unsichtbar.

28. April. (20 Min.). Schwaches continuirliches Spectrum, ultraviolette Linien unsichtbar.

29. April. (25 Min.). Vollständig wie am II. und 2 I. bis 22: April, ultraviolette Linie sehr deutlich, continuirliches. Spectrum sehr matt, Nebelline die hellste.

Aus diesen ganz flüchtigen Beschreibungen ist zu ersehen, dass ich zwei verschiedene Spectra - bei einem. das continuirliche, bei dem anderen das Gasspectrum über wiegend - beobachtet habe, welche periodisch wiederkehren.

Das continuirliche Spectrum habe ich sehr deutlich am 3r. März, 8., 18., 27. April und das Gasspectrum am 6. I I., 2 I., 22., 25., 29. April beobachtet. Es scheint, dass. das continuirliche Spectrum eine Periode von circa 9 Tagen hat und der Stern längere Zeit in dem Zustande verweilt, welcher dem Gasspectrum entspricht. Leider verhinderte das ungünstige Wetter besser ausreichendes Material zu sammeln, und jetzt bei dem tiefen Stand der Sterne ist wenig Hoffnung. vorhanden, die Perioden weiter verfolgen zu können.

Herény, Astrophysikalisches Observatorium, Igor Mai I.

Eugen v. Gothard.

\section{Beobachtungen des Cometen 1901 a.}

(Telegramm von Herrn P. Baracchi, Director der Sternwarte in Melbourne).

\begin{tabular}{|c|c|c|c|c|c|c|c|}
\hline Beob. & \multicolumn{3}{|c|}{1901} & RA. app. & \multicolumn{3}{|c|}{ PD. app. } \\
\hline Melbourne & April & $27 \cdot 355$ & Gr. m. t. & $15^{\circ} \times 3^{\prime}$ & $88^{\circ} 23^{\prime}$ & & uncertain \\
\hline$»$ & & 30.850 & $"$ & $4^{6} \quad 15$ & 9042 & & $»$ \\
\hline Tebbutt & Mai & 2.8380 & $\gg$ & $\begin{array}{lll}53 & 47 & 37\end{array}$ & 9035 & $29^{\prime \prime}$ & \\
\hline » & & 4.8426 & $»$ & $603^{8}$ & 908 & 12 & \\
\hline " & & 5.8572 & 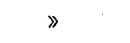 & $63 \quad 4^{6} \quad 25 \cdot 5$ & 8948 & 52 & \\
\hline
\end{tabular}

Die Melbourner Beobachtung vom 27. April steht in Widerspruch mit den Ortsbestimmungen am Cap und ist wohl zu verwerfen.

Die in London erscheinende »Daily Mail» enthält folgende, mir von dem Hamburger Vertreter derselben freund. lichst mitgetheilte Notiz über den Schweif des Cometen:

»A telegram has been received in London from the observatory at Mauritius stating that a photograph of the new comet has been secured. Three distinct tails are visible, the length of the longest being 15 degrees, or thirty times. the diameter of the moon. The comet was travelling north-west. «

$K r$.

Berichtigung zu AG. Leipzig II Zone $+5^{\circ}$ bis $+10^{\circ}$. Bei dem Stern Nr. 6 r9 I ist für die Declination statt: $6^{\circ} 47^{\prime}$ i2".6 zu setzen: $6^{\circ} 4^{8}$ i $2^{\prime \prime} 6$. B. Peter.

Inhalt zu Nr. 3713. 1.v. Benko. Mikrometer-Beobachtungen. 257. - I.v. Benko. Beobachtungen von Sternbedeckungen durch den Mond I 899-1900. 26I. - I. v. Benko. Beobachtung der Sonnenfinsterniss am 28. Mai 1900. 263. - I. v. Benko. Beobachtung der Saturnbedeckung am I3. Juni I900. 265.- L. Weinek. Zur Leonidenerscheinung I900. 265. - 7. Pidoux. Positions de petites Planètes. 267. - F. Holetschek. Beobachtungen des Cometen 1900 II. 267. - E. v. Gothard. Periodische Veränderungen im Spectrum der Nova Persei. 269. - Beobachtungen des Cometen 1901 a. 27I. - Berichtigung. $27 \mathrm{I}$.

Geschlossen Igor Mai r3. Herausgeber: H. Kreutz. Druok von C. S chaidt. Expedition: Kiel, Niemanusweg Io3. 